ACUTE LUNG INJURY

\title{
In vitro and in vivo effects of salbutamol on neutrophil function in acute lung injury
}

\author{
G D Perkins, N Nathani, D F McAuley, F Gao, D R Thickett
}

Thorax 2007;62:36-42. doi: 10.1136/thx.2006.059410

Background: Intravenous salbutamol (albuterol) reduces lung water in patients with the acute respiratory distress syndrome (ARDS). Experimental data show that it also reduces pulmonary neutrophil accumulation or activation and inflammation in ARDS.

Aim: To investigate the effects of salbutamol on neutrophil function.

Methods: The in vitro effects of salbutamol on neutrophil function were determined. Blood and

See end of article for authors' affiliations

......................

Correspondence to: G D Perkins, Lung Investigation Unit, Nuffield

House, Queen Elizabeth

Hospital, University of Birmingham, Edgbaston Birmingham B15 2TT, UK; gavin.perkins@virgin.net

Revised 29 July 2006

Accepted 1 August 2006

Published Online First

23 August 2006 bronchoalveolar lavage (BAL) fluid were collected from 35 patients with acute lung injury (ALI)/ARDS, 14 patients at risk from ARDS and 7 ventilated controls at baseline and after 4 days' treatment with placebo or salbutamol (ALI/ARDS group). Alveolar-capillary permeability was measured in vivo by thermodilution (PiCCO). Neutrophil activation, adhesion molecule expression and inflammatory cytokines were measured. Results: In vitro, physiological concentrations of salbutamol had no effect on neutrophil chemotaxis, viability or apoptosis. Patients with ALI/ARDS showed increased neutrophil activation and adhesion molecule expression compared with at risk-patients and ventilated controls. There were associations between alveolarcapillary permeability and BAL myeloperoxidase $(r=0.4, p=0.038)$ and BAL interleukin $8(r=0.38$, $p=0.033$ ). In patients with ALI/ARDS, salbutamol increased numbers of circulating neutrophils but had no effect on alveolar neutrophils.

Conclusion: At the onset of ALI/ARDS, there is increased neutrophil recruitment and activation. Physiological concentrations of salbutamol did not alter neutrophil chemotaxis, viability or apoptosis in vitro. In vivo, salbutamol increased circulating neutrophils, but had no effect on alveolar neutrophils or on neutrophil activation. These data suggest that the beneficial effects of salbutamol in reducing lung water are unrelated to modulation of neutrophil-dependent inflammatory pathways.
A cute lung injury (ALI) and the acute respiratory distress syndrome (ARDS) are major causes of respiratory failure in the critically ill patient. ${ }^{1}$ Although controversy still exists regarding the role of neutrophils in all causes of $\mathrm{ALI}^{2}$ a recent systematic review of laboratory and clinical studies concluded that neutrophils played a central part in most cases. ${ }^{3}$ Pathophysiologically, ARDS is characterised by intense inflammation in the alveolar space, with a predominance of neutrophils. Analysis of bronchoalveolar lavage (BAL) fluid from patients with ARDS has shown increased numbers of activated neutrophils in the early stages of ARDS. ${ }^{4}$ The number of neutrophils in BAL fluid relates to the severity of lung injury, ${ }^{6}$ and the persistence of neutrophils is associated with increased mortality. ${ }^{5}$ A study examining BAL fluid from patients with ARDS showed a positive correlation between neutrophil myeloperoxidase (MPO) and oxidatively modified amino acids, suggesting an association between pulmonary neutrophil activation and oxidative protein damage. ${ }^{7}$ Animal studies have shown that neutrophil-mediated lung injury reduces alveolar fluid clearance, preventing the resolution of non-cardiogenic pulmonary oedema. ${ }^{8}$ Neutrophil elastase inhibitors in animal models limit the degree of lung injury caused by ischaemia reperfusion ${ }^{9}$ and lipopolysaccharide (LPS), ${ }^{10}$ although a recent multicentre clinical trial of the elastase inhibitor Sivelestat failed to improve outcome in a heterogeneous group of patients with ARDS. ${ }^{11}$

$\beta_{2}$-agonists have several inhibitory effects on neutrophil function. ${ }^{12}$ In animal models of acute lung injury, $\beta_{2}$-agonists reduce pulmonary neutrophil sequestration. ${ }^{13}{ }^{14}$ In vitro $\beta_{2}$ agonists reduce the production of oxygen free radicals from neutrophils and other inflammatory cells ${ }^{15}$ and reduce inflammatory cytokine production. ${ }^{17}$ In humans, inhaled salmeterol (long-acting $\beta_{2}$-agonist) inhibited LPS-induced neutrophil influx, degranulation and tumour necrosis factor $(\mathrm{TNF}) \alpha$ release. $^{18}$ These experimental findings suggest that treatment with a $\beta_{2}$-agonist could have a favourable effect in ARDS by reducing neutrophilic inflammation.

We recently conducted a randomised controlled trial in humans with ARDS ( $\beta$-agonist lung injury trial (BALTI-1) study), and showed that intravenous salbutamol (albuterol) markedly reduced extravascular lung water probably through up regulation of alveolar fluid clearance. ${ }^{19}$ However, the reduction in lung water was not seen until $48 \mathrm{~h}$ after the initiation of treatment, suggesting some effects in addition to alveolar fluid clearance. The objective of this study was to investigate whether salbutamol modulates neutrophil function and neutrophilic inflammation at physiologically relevant doses in patients with ALI. The second objective was to investigate the relationship between neutrophilic inflammation and alveolar capillary permeability in patients with ARDS.

\section{MATERIALS AND METHODS}

In vitro studies

Under-agarose chemotaxis, cell viability and apoptosis

Neutrophils from non-smoking, healthy controls were purified from peripheral blood by discontinuous Percoll density gradients. ${ }^{20}$ The effect of salbutamol on the chemotactic activity of purified neutrophils was measured using the under-agarose

Abbreviations: $A L I$, acute lung injury; ARDS, acute respiratory distress syndrome; BAL, bronchoalveolar lavage; FMLP, N-formyl-L-leucinmethionyl-L-phenylalanine; LPS, lipopolysaccharide; TNF, tumour necrosis factor 
method. ${ }^{21}$ The freshly harvested neutrophils were resuspended at $5 \times 10^{7}$ cells $/ \mathrm{ml}$ in RPMI (Sigma, UK) 1640 culture medium or $10^{-5}-10^{-10} \mathrm{M}$ salbutamol in RPMI (with or without propranolol $10^{-4} \mathrm{M}$ ) before seeding in $10-\mu \mathrm{l}$ aliquots in the central wells of a freshly prepared agarose plate. In all, $10 \mu \mathrm{l}$ of RPMI was placed in each of the inner wells and $10 \mu \mathrm{l}$ of chemoattractant $\left(10^{-7} \mathrm{~N}\right.$-formyl-L-leucin-methionyl-L-phenylalanine (FMLP, Sigma, UK) in RPMI) in each of the outer wells. Negative controls contained RPMI in the inner and outer wells. The plates were incubated at $37^{\circ} \mathrm{C}\left(5 \% \mathrm{CO}_{2}\right)$ for $2 \mathrm{~h}$ and then flooded with methanol for overnight fixation of the cells. The gel was carefully removed and the plates washed under slow running tap water. The plates were then stained with Gram stain and left to dry. For each well, the chemotactic and chemokinetic responses were read using an eyepiece graticule. The overall response to the chemoattractant (chemotactic differential) was calculated by subtracting the chemokinetic response from the chemotactic response.

Purified neutrophils, suspended in RPMI at a concentration of $1 \times 10^{6} \mathrm{ml}$, were incubated with $10^{-5}$ and $10^{-7} \mathrm{M}$ salbutamol or RPMI control for $2 \mathrm{~h}$ at $37^{\circ} \mathrm{C}$ in humidified $5 \% \mathrm{CO}_{2}$. Cell proliferation was assessed by adding $20 \mu \mathrm{l}$ of CellTiter 96 AQueous one solution (Promega, Southampton, UK) to $100 \mu \mathrm{l}$ of cell suspension in a 96-well culture plate (Nunc). The reaction was allowed to proceed for $2 \mathrm{~h}$ at $37^{\circ} \mathrm{C}$ in $5 \% \mathrm{CO}_{2}$. The cell titre solution contains a tetrazolium compound that is metabolised by healthy cells to a formazan product, the absorbance of which was read at $495 \mathrm{~nm}$ on an MRX-II 96well plate reader (Dynex Technologies, Sussex, UK).

Purified neutrophils, suspended in RPMI with 10\% heatinactivated fetal calf serum (Life Technologies) and 100 units/ $\mathrm{ml}$ penicillin and $100 \mathrm{mg} / \mathrm{ml}$ streptomycin (Sigma, Poole, UK) at a concentration of $1 \times 10^{6} \mathrm{ml}$, were incubated with $10^{-5}$ and $10^{-7} \mathrm{M}$ salbutamol or RPMI control for $18 \mathrm{~h}$ at $37^{\circ} \mathrm{C}$ in humidified $5 \% \mathrm{CO}_{2}$. Preliminary experiments showed that $18 \mathrm{~h}$ was the optimal time point for assessing apoptosis. The number of normal and apoptotic neutrophils were determined by morphological patterns. After centrifugation, samples were stained using a commercial May-Grunwald Giemsa stain (DiffQuick, Baxter Healthcare Product, Deerfield, Illinois, USA). The percentage apoptotic cells compared with total number of cells were calculated. The morphological results were confirmed using flow cytometry and the annexin V/propidium iodine apoptosis kit (Dako, Carpinteria, California, USA). The percentages of live (annexin and propidium iodine negative) and early apoptotic (annexin positive, prodipium iodine negative) or late apoptotic/dead (annexin positive, prodipium iodine positive) cells were calculated.

\section{Clinical study}

Mechanically ventilated adult patients enrolled in the BALTI- 1 study were eligible for inclusion. ${ }^{19}$ This study recruited patients within $48 \mathrm{~h}$ of the onset of ALI and ARDS and randomised them to 7 days of treatment with intravenous salbutamol $(15 \mu \mathrm{g} / \mathrm{kg} / \mathrm{h})$. ALI was defined according to the American European Consensus Conference definition ${ }^{22}$ as the acute onset of respiratory failure with a arterial oxygen tension:fractional oxygen tension $\left(\mathrm{PaO}_{2}: \mathrm{FiO}_{2}\right)$ ratio of $<300 \mathrm{~mm} \mathrm{Hg}$ and bilateral infiltrates on the chest radiograph in the absence of clinical evidence of left atrial hypertension. ARDS was considered present when the $\mathrm{PaO}_{2}: \mathrm{FiO}_{2}$ ratio was $<200 \mathrm{~mm} \mathrm{Hg}$. The exclusion criteria were as follows: age $<18$ years; participation in other intervention trials; severe obstructive airway disease requiring nebulised or intravenous $\beta_{2}$-agonist; treatment with $\beta$-blockers within $48 \mathrm{~h}$; neutrophil count $<0.3 \times 10^{9} \mathrm{l}$; brain stem death; treatment withdrawal within $24 \mathrm{~h}$; immunosuppression (steroids $>20 \mathrm{mg} /$ day, chemotherapy or other immunosuppressive agents within 2 weeks); lobectomy/ pneumonectomy; burns $>40 \%$ body surface area; assent declined from the next of kin.

Patients with identifiable risk factors for ALI/ARDS, but who, at the time of recruitment had not met the criteria for

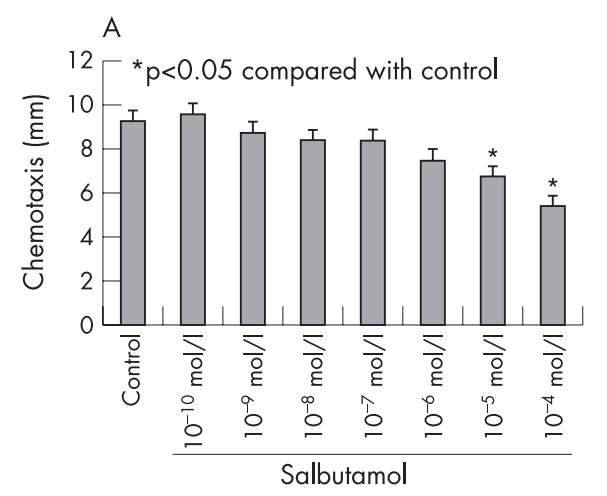

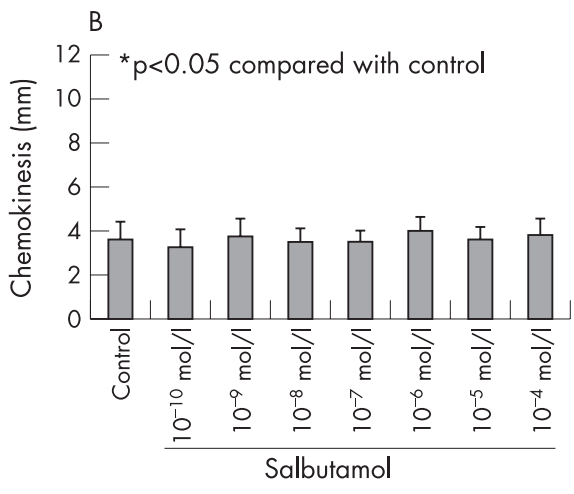

Figure 1 (A) Salbutamol $10^{-5} \mathrm{M}$ and $10^{-4} \mathrm{M}$ significantly reduced neutrophil chemotaxis towards the chemotractant stimulus FMLP. (B) No effect was seen on neutrophil chemokineses. (C) The effect of salbutamol on neutrophil chemotaxis was blocked by incubation with $10^{-4} \mathrm{M}$ propranolol. (D) Salbutamol $\left(10^{-6}\right.$ and $10^{-4} \mathrm{M}$ ) had no effect on the rate of apoptosis at $18 \mathrm{~h}$ (apoptosis determined by morphology (grey bars) and flow cytometry (black bars). Data shown are mean (standard error) from six experiments. ${ }^{*} \mathrm{p}<0.05$ compared with control.
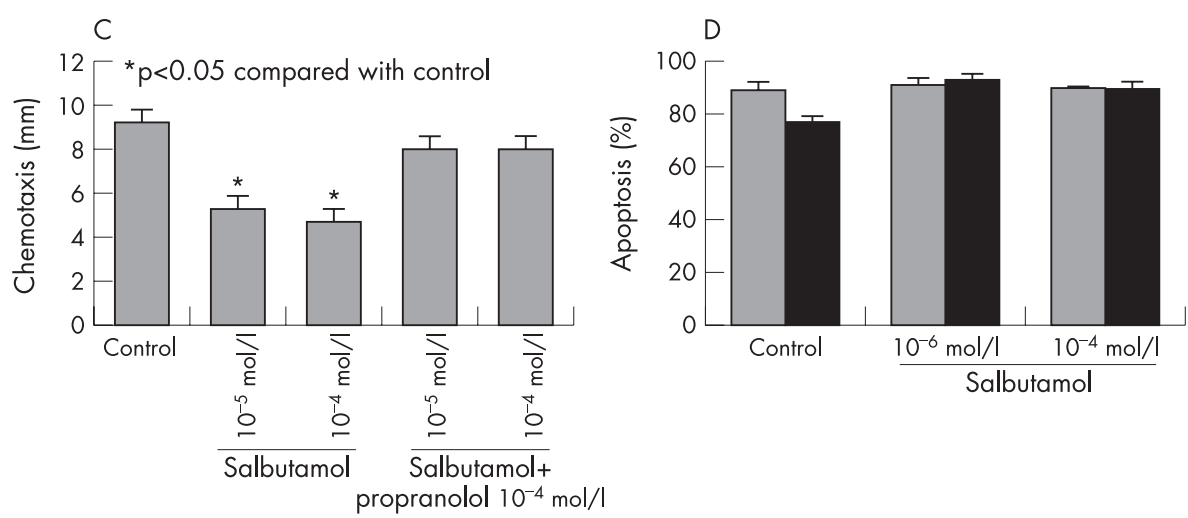
ALI/ARDS, were included as an at risk-population. Nonsmoking, age-matched ventilated patients undergoing elective surgery were recruited as controls.

\section{Biological sample collection and processing}

BAL using $150 \mathrm{ml}$ cold saline was performed immediately after recruitment in at-risk patients and ventilated controls and at baseline and on day 4 in patients with ALI/ARDS. BAL fluid was kept on ice until transferred to the laboratory for processing. Blood was simultaneously collected in lithium heparin tubes (Becton-Dickinson, Plymouth, UK) and placed immediately on ice until processed.

The BAL fluid was filtered through coarse surgical gauze to remove mucus and other debris. The fluid was then centrifuged at $500 \mathrm{~g}$ for $5 \mathrm{~min}$ in a centrifuge prechilled to $4^{\circ} \mathrm{C}$. The supernatant was removed and immediately frozen to $-80^{\circ} \mathrm{C}$ and stored for subsequent analysis. The cell pellet was resuspended in $10 \mathrm{ml}$ phosphate-buffered saline supplemented with $1 \%$ human serum albumin. Total cell count was determined using a haemocytometer. Cell viability was measured by the ability of live cells to exclude Trypan Blue. Cell purity was measured on a cytospin preparation stained with DiffQuick (Baxter, Berkshire, UK) as described previously . ${ }^{23}$

\section{Patient characteristics}

Patient demographic characteristics were recorded at baseline. The acute physiology and chronic health evaluation II (APACMEII) and simplified acute physiology score II (SAPSII) scores, and predicted intensive care unit mortality were recorded as global markers of disease severity. ${ }^{24}$ The Murray Lung Injury Score and $\mathrm{PaO}_{2}: \mathrm{FiO}_{2}$ ratio were collected as markers of the severity of lung injury.

\section{Alveolar-capillary permeability}

The single indicator transpulmonary thermodilution system (PiCCO; Pulsion Medical Systems, Munich, Germany) was used to calculate an in vivo alveolar-capillary permeability index. The permeability index was derived from the ratio of extravascular lung water to pulmonary blood volume. Previous studies have shown that this index can separate cardiogenic (low permeability) and inflammatory (high permeability) causes of pulmonary oedema. ${ }^{25}$

\section{Flow cytometry analysis}

Flow cytometry was performed using the whole-blood technique. ${ }^{26}$ Aliquots of $100 \mu \mathrm{l}$ of whole blood or BAL cells (suspended in $1 \%$ human serum albumin (Sigma Chemicals) at a final concentration of $1 \times 10^{6} / \mathrm{ml}$ ) were fixed by adding $100 \mu \mathrm{l}$ of $1 \%$ paraformaldehyde for $15 \mathrm{~min}$. Immunofluorescent staining was performed by adding fluorescin isothiocyante isomer-conjugated monoclonal antibodies directed against the following: CDllB (IgGl, Dako, Ely, UK); CDl8 (IgGl, Dako); CD49 (IgGl,
Serotec, Oxford, UK), CD64 (IgGl, Serotec) and L-selectin, IgGl, (Becton-Dickinson). Stimulated neutrophil L-selectin expression was determined by incubating whole blood with FMLP $\left(10^{-6} \mathrm{M}\right)$ for $15 \mathrm{~min}$ before fixation. Controls were included using isotype-matched, irrelevant antibodies to human IgGl (Dako). After incubation with the appropriate monoclonal antibody for $30 \mathrm{~min}$ at room temperature (protected from light), $1.5 \mathrm{ml}$ of FACS brand lysing solution (Becton-Dickinson) was added to each tube for $5 \mathrm{~min}$. The cells were then washed twice with $1.5 \mathrm{ml}$ of wash buffer ( $500 \mathrm{ml}$ phosphate-buffered saline, $0.1 \mathrm{~g}$ sodium azide (Sigma Chemicals), $5 \mathrm{~g}$ bovine serum albumin (Sigma)). After the second wash, the cell pellet was resuspended in $500 \mu \mathrm{l}$ of $1 \%$ paraformaldehyde. Samples were analysed on a BectonDickinson 440 flow cytometer. The neutrophil cell populations were identified and gated from the forward and side light scatter. The median intensity of fluorescence for cells labelled with specific antibody was determined relative to the median intensity of fluorescence for cells labelled with the non-specific isotype control antibody.

\section{Tumor necrosis factor $\alpha$, interleukin 8, MPO and} salbutamol assays

Tumor necrosis factor $\alpha(\mathrm{TNF} \alpha)$ and interleukin (IL) 8 levels in BAL fluid were measured using a commercially available ELISA (R\&D systems, Abingdon, UK). BAL MPO was measured using the chromogenic substrate assay as described previously. ${ }^{23}$ Salbutamol levels were measured using a commercially available ELISA (Biox diagnostics, Jemelle, Belgium).

\section{Statistical analysis}

The study was powered to detect a $60 \%$ reduction in alveolar neutrophil sequestration based on recent data showing that $\beta_{2^{-}}$ agonists reduce pulmonary neutrophil sequestration in human volunteers exposed to LPS by $60 \% .{ }^{18}$ We calculated that nine patients would need to be recruited in each arm to detect this difference with $80 \%$ power at a significance level of 0.05 . Differences between groups were examined by analysis of variance or the Kruskal-Wallis test. Where significant differences were identified, Tukey's test or the Mann-Whitney U test was used to further examine the differences. Repeated measures of neutrophil counts were non-parametric and analysed by Friedman's repeated measures test. Linear associations were tested using Pearsons correlation test. Data are expressed as mean (standard deviation) unless otherwise stated; $\mathrm{p}<0.05$ was considered significant.

\section{RESULTS}

\section{In vitro studies}

Neutrophil migration towards the chemotractant stimulus FMLP was greater than that towards the RPMI control (11.6 $\mathrm{mm}(1.2)$ versus $3.6 \mathrm{~mm}(0.2), \mathrm{p}<0.001)$. Salbutamol at

Table 1 Baseline demographics for patients at risk from acute respiratory distress syndrome and patients with established acute respiratory distress syndrome

\begin{tabular}{lllll}
\hline & $\begin{array}{l}\text { ARDS } \\
(\mathbf{n}=\mathbf{3 5})\end{array}$ & $\begin{array}{l}\text { At risk } \\
(\mathbf{n}=19)\end{array}$ & $\mathbf{p}$ Value & $\mathbf{9 5 \% \mathrm { Cl }}$ \\
\hline P:F ratio $\mathrm{mm} \mathrm{Hg}(\mathrm{kPa})$ & $106(92)(33.5(12.1))$ & $155(92)(14.9(5.8))$ & $<0.001$ & $95-185(12.5$ to 24.4$)$ \\
Lung injury score & $2.9(0.6)$ & $1.2(0.3)$ & 0.001 & 1.3 to 1.9 \\
SOFA score & $13.4(3.7)$ & $6.7(4.3)$ & 0.001 & 4.1 to 9.2 \\
APACHE II & $23.4(6.9)$ & $21.7(8.9)$ & 0.312 & -2.1 to 6.5 \\
SAPS II & $52.0(15.7)$ & $54.8(12.8)$ & 0.550 & -12.0 to 6.4 \\
Direct lung injury & $13(37 \%)$ & $6(32 \%)$ & 0.771 & \\
Indirect lung injury & $22(63 \%)$ & $13(68 \%)$ & & \\
\hline
\end{tabular}

APACHE, acute physiology and chronic health evaluation; ARDS, acute respiratory distress syndrome; SAPS, simplified acute physiology score; SOFA, sequential organ failure assessment. 
$10^{-4}$ and $10^{-5} \mathrm{M}$ significantly reduced neutrophil chemotaxis (fig lA). There was no effect on random neutrophil chemokinesis (fig 1B). The effect of salbutamol on neutrophil chemotaxis was abolished by propranolol (fig IC). Salbutamol $10^{-4}$ to $10^{-6} \mathrm{M}$ had no effect on cellular viability (data not shown) or neutrophil apoptosis (fig lD).

\section{Clinical studies}

Patient baseline demographics

Forty patients with ALI/ARDS were initially recruited, of whom 35 had BAL performed at baseline and were included in this study. Fourteen patients with risk factors for ARDS but who had not developed ARDS at the time of BAL formed the at risk group. Some of the data from the at risk group (BAL MPO levels and neutrophil counts) have been published previously. ${ }^{23}$ Seven ventilated controls were recruited. Table 1 shows the baseline data on severity of illness and aetiology of lung injury for the patients with ARDS and those at risk.

\section{Neutrophilic inflammation is present in the alveolar space in patients with ARDS}

Evidence of increased accumulation of neutrophils and neutrophilic inflammation was found in the BAL fluid of patients with ARDS. Total neutrophil count, MPO, TNF $\alpha$ and IL8 levels were raised in patients with ARDS compared with patients at risk from ARDS and normal ventilated controls (fig 2 (A-D)). We found significant, albeit weak, linear associations between the PiCCO alveolar-capillary permeability index and
MPO ( $\mathrm{r}=0.4, \mathrm{p}=0.038)$ and IL8 $(\mathrm{r}=0.37, \mathrm{p}=0.033)$ levels. Compared with normal controls, there was evidence of increased adhesion molecule expression CD11B, CD18, CD49, reduced L-selectin expression and increased neutrophil activation (CD64) on circulating and alveolar neutrophils (fig 3).

\section{In vivo effects of salbutamol}

From the initial group of patients randomised to salbutamol or placebo, follow-up samples of blood and BAL fluid were collected from 22 patients at day 4 ( 9 randomised to salbutamol and 13 to placebo). Salbutamol was detected by ELISA in the plasma of the treatment group at a concentration equivalent to $10^{-6} \mathrm{M}$ (range $250-495 \mathrm{ng} / \mathrm{ml}$ ). The ELISA did not perform reproducibly in the BAL matrix.

\section{Circulating neutrophils}

Treatment with intravenous salbutamol significantly increased the number of circulating neutrophils (fig 4). There was a trend towards reduced L-selectin expression on unstimulated neutrophils in the salbutamol group (placebo 55 (19) $v$ salbutamol 39 (30), $\mathrm{p}=0.18$ ). When neutrophils were stimulated ex vivo with FMLP, neutrophils from salbutamol-treated patients had significantly lower L-selectin expression (placebo 43 (15) $v$ salbutamol $22(21), 95 \%$ confidence interval (CI) 1.2 to 39 , $\mathrm{p}=0.038$; fig 5 ). We found no effect on integrin adhesion molecule expression measured at day 4 (CD11B, CD18 or CD49) or expression of the neutrophil activation marker CD64 (fig 3A).
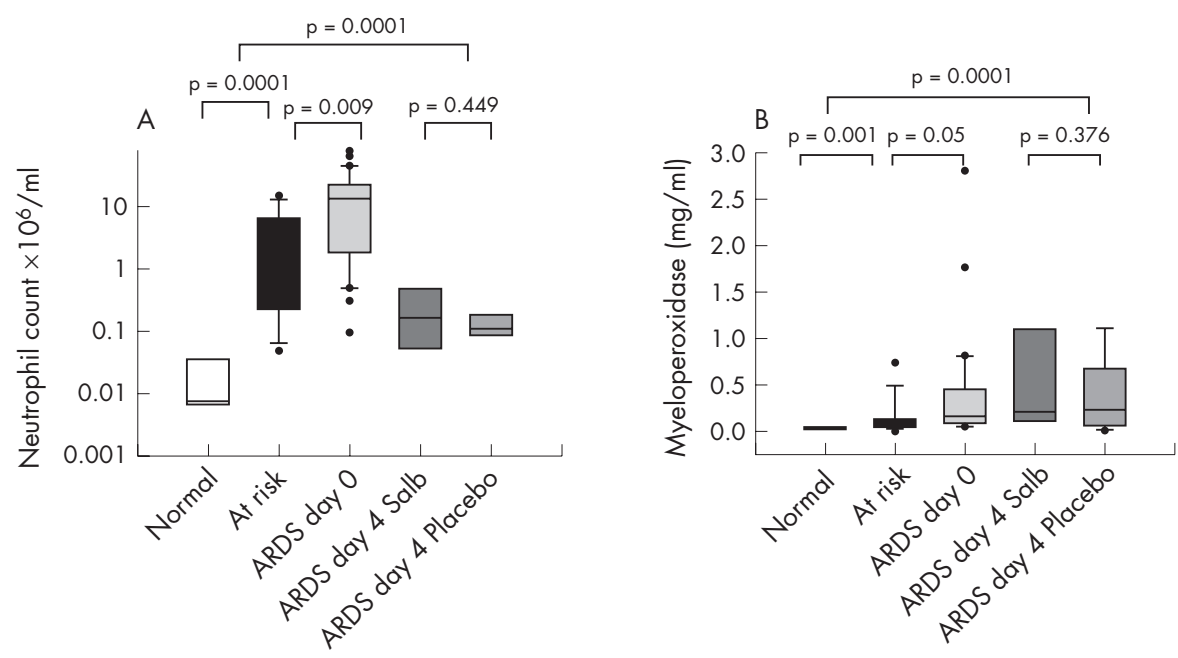

Figure 2 (A) Bronchoalveolar lavage neutrophil count, (B) myeloperoxidase, (C) tumour necrosis factor $\alpha(\mathrm{TNF} \alpha)$ and (D) interleukin (IL)8 in normals, patients at risk from acute lung injury (ALI)/acute respiratory distress syndrome (ARDS) and patients with ALI/ARDS at baseline and after 4 days of treatment with intravenous salbutamol (salb).
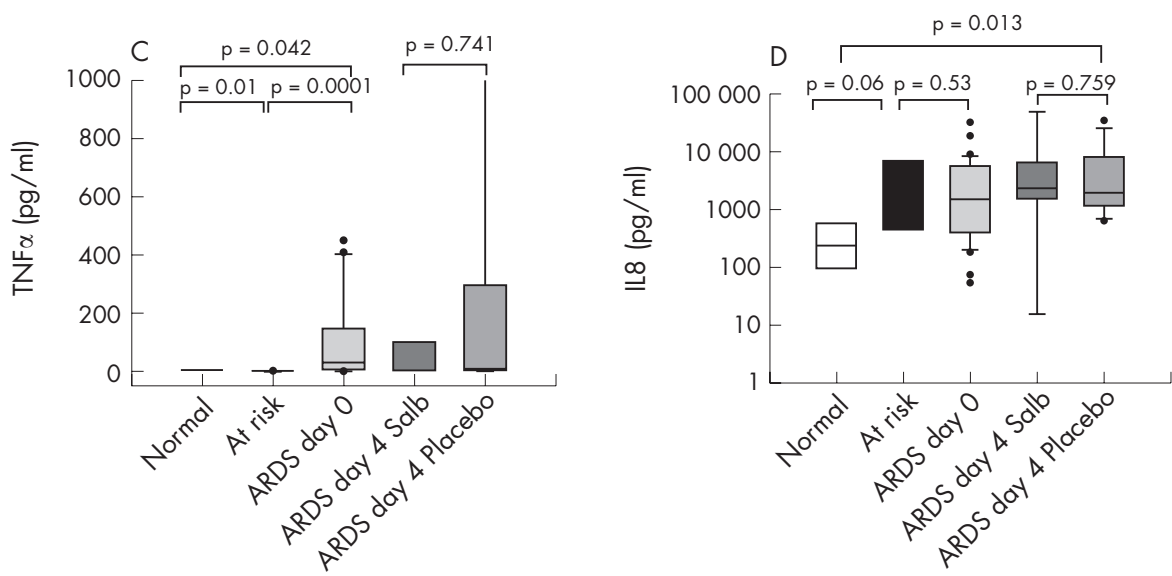


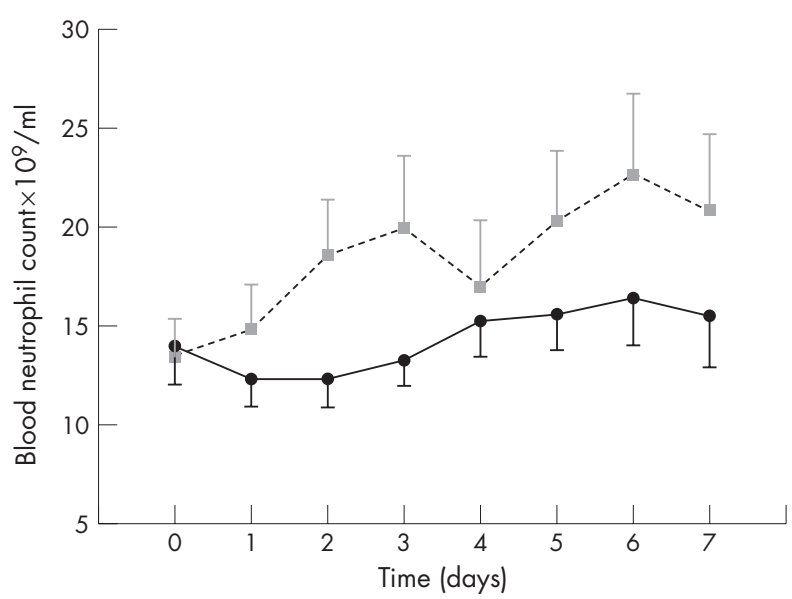

Figure 3 Treatment with intravenous salbutamol significantly increased circulating neutrophil count $(p=0.004)$. Data are shown as mean (SE) for salbutamol (squares/grey dotted line) and placebo (circles/solid line).

Pulmonary neutrophils and alveolar inflammation In contrast to the plasma compartment, there was no difference in BAL fluid neutrophil count between groups at day 4 (placebo $3.5(0.6) v$ salbutamol group 1.6 (1.7), $\mathrm{p}=0.4$; fig 2A). Adhesion molecule expression was also similar in the two groups and there was no difference in the expression of the neutrophil activation marker CD64 (fig 3B). We found no difference in L-selectin expression (unstimulated cells: placebo 3 (4) $v$ salbutamol 4 (6), $\mathrm{p}=0.753$; FMLP stimulated cells: placebo 1.7 (2) $v$ salbutamol $1.8(3), \mathrm{p}=0.9$ ). We also found no differences in myeloperoxidase (fig $2 \mathrm{~B}$ ), TNF $\alpha$ (fig 2C) or IL8 (fig 2D).

\section{DISCUSSION}

In a double-blind randomised placebo-controlled trial, we showed that sustained treatment with intravenous salbutamol considerably reduced extravascular lung water in humans with ALI/ARDS. ${ }^{19}$ In contrast with our initial hypothesis and supporting data from animal studies, ${ }^{27}$ the response was not evident until $48 \mathrm{~h}$ after the initiation of treatment. Our study sought to identify whether salbutamol was having an effect on pulmonary neutrophil accumulation and alveolar inflammation. Our study confirms previous observations that there is increased neutrophil accumulation and inflammation in the alveolar space in people with ALI/ARDS. The novel finding of a positive association between the intensity of neutrophilic infiltration and an in vivo measurement of alveolar-capillary permeability adds additional support to the hypothesis that the neutrophil is a key inflammatory mediator in ARDS. Contrary to in vitro, animal and healthy volunteer studies, we found no evidence that intravenous salbutamol modulated alveolar neutrophil accumulation, activation or markers of alveolar inflammation in humans with ALI/ARDS.

Studies investigating the in vitro effects of $\beta$-agonists on neutrophil chemotaxis have produced conflicting results, probably due to differences in the specific $\beta_{2}$-agonist tested, the dose used and the experimental conditions. Some studies showed a reduction in neutrophil chemotaxis, ${ }^{28}{ }^{29}$ whereas others reported a biphasic response with increased neutrophil chemotaxis at low concentrations of $\beta_{2}$-agonist and a reduction in chemotaxis at higher concentrations. ${ }^{30}$ Our study reports for the first time that the physiological concentration of salbutamol achieved in the plasma after an intravenous infusion of salbutamol $(15 \mu \mathrm{g} / \mathrm{kg} / \mathrm{h})$ in patients with ALI/ARDS is
$10^{-6} \mathrm{M}$. At this concentration, no effect on neutrophil chemotaxis was observed. Supraphysiological concentrations $\left(10^{-4}\right.$ and $\left.10^{-5} \mathrm{M}\right)$, however, reduced neutrophil chemotaxis through activation of the $\beta$-receptor. Moreover, physiologically relevant concentrations of salbutamol had no effect on neutrophil viability or the rate of spontaneous apoptosis measured by morphology and annexin V/propidium iodine staining.

The mechanisms regulating pulmonary neutrophil sequestration have been well characterised. Pulmonary neutrophil sequestration occurs within minutes of exposure to an inflammatory insult. ${ }^{31}{ }^{32}$ The insult causes an increase in neutrophil stiffness and reduction in deformability, ${ }^{33}$ leading to sequestration into the pulmonary capillaries, followed by emigration into the alveolar space. The process of neutrophil emigration occurs by at least two differentially regulated pathways: CDl1/18 adhesion molecule interactions determine the response to Gram-negative organisms, ILl $\alpha$ and phorbol 12myristate 13-acetate, whereas Gram-positive organisms, hyperoxia and the complement anaphylatoxins (C5a) seem to induce neutrophil emigration through a CDI1/18-independent pathway. ${ }^{34}$

Animal models of direct ${ }^{13}$ and indirect ${ }^{14}$ lung injury have shown that pretreatment with intravenous $\beta_{2}$-agonists reduces pulmonary neutrophil sequestration by $30 \%$. In normal human volunteers, in a placebo-controlled trial, treatment with $300 \mu \mathrm{g}$ inhaled salbutamol prevented platelet-activating factorinduced pulmonary sequestration of radiolabelled neutrophils. ${ }^{35}$ Pretreatment with salmeterol similarly inhibited LPS-induced neutrophil influx, neutrophil degranulation (myeloperoxidase) and TNF $\alpha$ release in human volunteers. ${ }^{18}$ Reduced neutrophilendothelial adhesion, through the down regulation of neutrophil integrin adhesion molecule expression (CD11B/18) seen with $\beta_{2}$-agonists may partly explain this finding. ${ }^{36}$

In our study, intravenous salbutamol considerably increased circulating neutrophil count but had no apparent effect on alveolar neutrophil counts. The increase in circulating neutrophils induced by $\beta$-agonists is thought to be due to detachment of cells from the marginating neutrophil pools rather than mobilisation of neutrophils from the bone marrow. ${ }^{37}$ Neutrophil L-selectin expression may be important in this effect because of a dominant role in the initial slowing, margination and rolling behaviour of neutrophils over the endothelim at the postcapillary venule in the systemic circulation. ${ }^{38}$ The shedding of Lselectin from the neutrophil surface allows neutrophils to break free from the vascular endothelium and return to the circulation. The mobilisation of neutrophils was not, however, associated with any difference in the degree of organ dysfunction between the two groups. ${ }^{19}$

In contrast to the systemic circulation, where neutrophil sequestration usually occurs at the post-capillary venule, neutrophil migration in the lung occurs at the pulmonary capillaries, without dependence on L-selectin-mediated rolling. ${ }^{39}$ In our study, we found a trend towards reduced L-selectin expression on circulating neutrophils in patients treated with salbutamol. When the neutrophils were maximally stimulated with FMLP, neutrophils primed by treatment with salbutamol showed a notably greater reduction in L-selectin expression than those treated with placebo. This finding is consistent with the observation that isoprotenerol reduces neutrophil L-selectin expression in human volunteers. ${ }^{40}$ Enhanced L-selectin shedding would increase neutrophil mobilisation from the systemic circulation and may explain the finding of increased circulating neutrophils. By contrast, the absence of a measurable effect of salbutamol on integrin adhesion molecule expression relevant to pulmonary neutrophil emigration may explain the observed lack of effect on pulmonary neutrophil sequestration. 

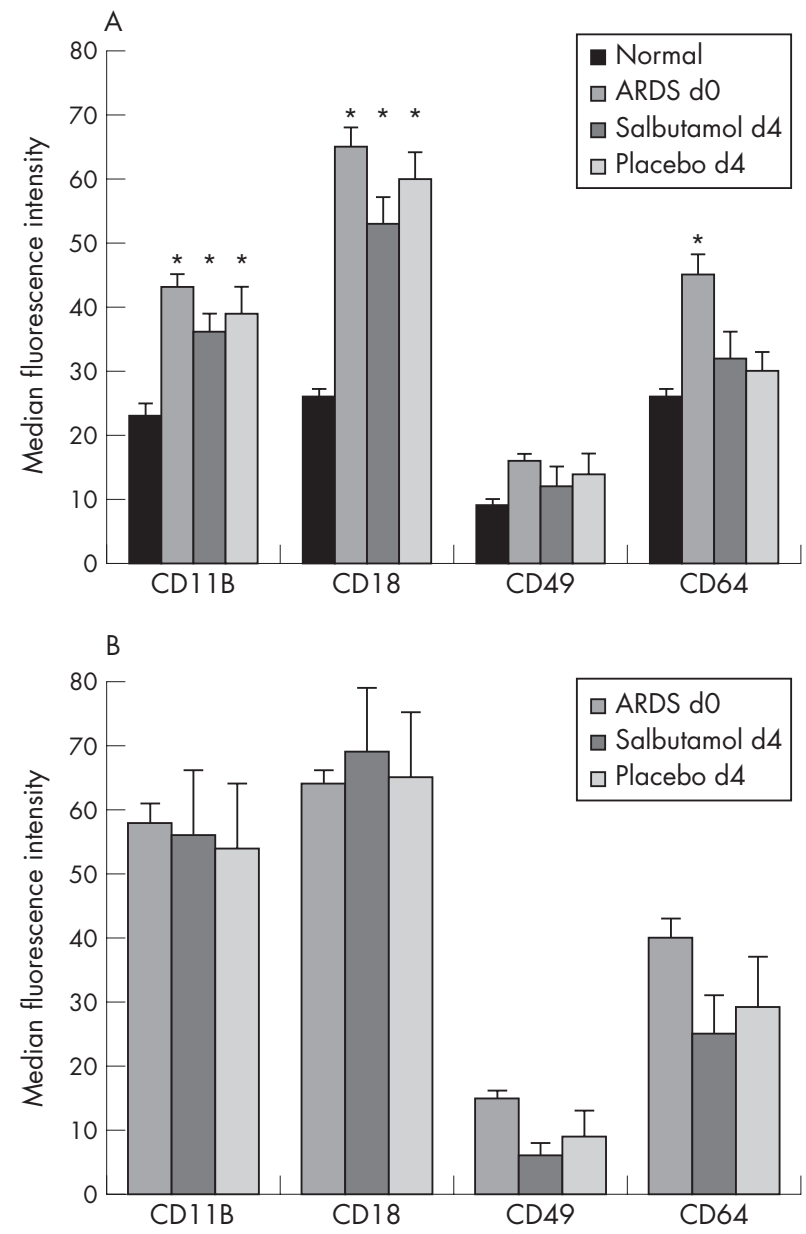

Figure 4 (A) Circulating and (B) alveolar neutrophil adhesion molecule (CD1 1b, CD18 and CD49) and activation maker (CD64) expression in normal volunteers and patients with acute lung injury (ALI)/acute respiratory distress syndrome (ARDS) at baseline and after 4 days of treatment with intravenous salbutamol. Data shown are mean (SE).

There are several other potential explanations for the absence of a measurable effect of salbutamol in vivo on alveolar neutrophil accumulation and inflammation. Firstly, pulmonary neutrophil emigration occurs within minutes of exposure to the toxic insult. ${ }^{32}$ In the previous studies that reported a reduction in pulmonary neutrophil sequestration with $\beta$ agonists, the drug was administered before the inflammatory insult. ${ }^{13} 141835$ In our study, salbutamol was given many hours after the initial insult, potentially therefore too late to have an effect on pulmonary neutrophil recruitment. Although the study was relatively small, it is similar in size to previous studies, ${ }^{13} 141835$ and was powered to detect a treatment effect of a magnitude similar to that observed in previous studies. We cannot, however, exclude that a smaller treatment effect would not have been observed with greater numbers, although the clinical relevance of smaller changes is unclear. Further, multiple signalling pathways enhance neutrophil emigration in patients with ARDS, which may differ depending on the aetiology of ARDS. Our study was not powered to investigate the effects of salbutamol on neutrophil inflammation because of different aetiologies, and so cannot exclude an effect in some causes of ARDS. Finally, alveolar neutrophil recruitment was only studied at baseline and 4 days after the initiation of treatment (up to 6 days after the onset of ARDS). In both treatment and placebo groups, neutrophil counts were

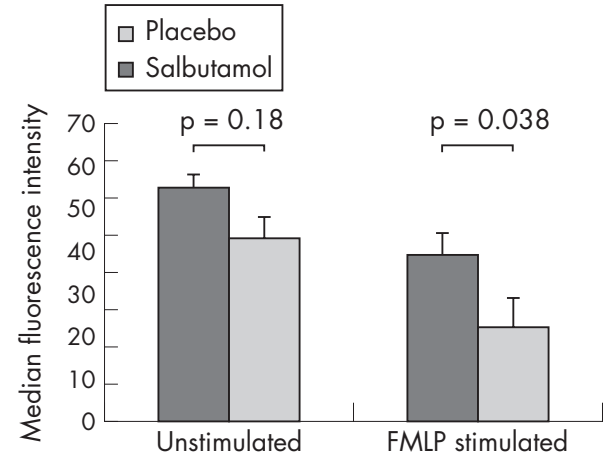

Figure 5 Effect of systemic treatment with intravenous salbutamol on resting and N-formy--Lleucin-methionyl-L-phenylalanine (FMLP) stimulated neutrophil L-selectin expression. Data shown are mean (SE).

markedly lower on day 4 than at the point of initial recruitment. Therefore, we cannot exclude that salbutamol may have had an effect earlier in the course of the disease, which by virtue of the timing of alveolar sampling, may have been missed.

\section{CONCLUSION}

At the onset of ALI/ARDS, there is increased pulmonary neutrophil recruitment and activation in addition to positive correlations between IL8, myeloperoxidase and alveolar-capillary permeability, suggesting an association between neutrophil activation and the development of lung injury. In vitro, physiological concentrations of salbutamol failed to show an effect on neutrophil chemotaxis, viability or apoptosis. Treating patients with ALI/ARDS with intravenous salbutamol increased the number of circulating neutrophils, but had no effect on alveolar neutrophil numbers or on neutrophil activation or alveolar inflammation. The beneficial effects of salbutamol in reducing extravascular lung water seem to be unrelated to the modulation of neutrophilic inflammatory pathways.

\section{Authors' affiliations}

G D Perkins, F Gao, Intensive Care Unit, Birmingham Heartlands Hospital, Birmingham, UK

N Nathani, D R Thickett, Lung Investigation Unit, Queen Elizabeth Hospital, University of Birmingham, Birmingham, UK

D F McAuley, Department of Intensive Care Medicine, Queen's University of Belfast, Belfast, UK

Funding: This study was supported by a grant from the West Midlands Intensive Care Society, Queen Elizabeth Hospital, Birmingham, UK.

Competing interests: GDP, DFMcA and DRT have received payment to attend scientific meetings and have given talks for pharmaceutical companies that manufacture $\beta$ - agonists (Astra Zeneca, GlaxoSmithKline).

Ethical approval: This study was approved by the East Birmingham Research Ethics Committee (Reference SJR/LMH/0522).

\section{REFERENCES}

1 Rubenfeld GD, Caldwell E, Peabody E, et al. Incidence and outcomes of acute lung injury. N'Engl J Med 2005;353:1685-93.

2 Laufe MD, Simon RH, Flint A, et al. Adult respiratory distress syndrome in neutropenic patients. Am J Med 1986;80:1022-6.

3 Abraham E. Neutrophils and acute lung injury. Crit Care Med 2003;31:S195-9.

4 Chollet-Martin S. Polymorphonuclear neutrophil activation during the acute respiratory distress syndrome. Intens Care Med 2000;26:1575-7.

5 Steinberg KP, Milberg JA, Martin TR, et al. Evolution of bronchoalveolar cell populations in the adult respiratory distress syndrome. Am J Respir Crit Care Med 1994; 150:113-22.

6 Sinclair DG, Braude S, Haslam PL, et al. Pulmonary endothelial permeability in patients with severe lung injury. Clinical correlates and natural history. Chest 1994;106:535-9.

7 Lamb NJ, Gutteridge JM, Baker C, et al. Oxidative damage to proteins of bronchoalveolar lavage fluid in patients with acute respiratory distress syndrome: 
evidence for neutrophil-mediated hydroxylation, nitration, and chlorination [see comments]. Crit Care Med 1999;27:1738-44.

8 Pittet JF, Lu LN, Morris DG, et al. Reactive nitrogen species inhibit alveolar epithelial fluid transport after hemorrhagic shock in rats. J Immunol 2001;166:6301-10.

9 Carden D, Xiao F, Moak C, et al. Neutrophil elastase promotes lung microvascular injury and proteolysis of endothelial cadherins. Am J Physiol 1998;275: H385-92.

10 Sakamaki $\mathbf{F}$, Ishizaka A, Urano T, et al. Effect of a specific neutrophil elastase inhibitor, ONO-5046, on endotoxin-induced acute lung injury. Am J Respir Crit Care Med 1996;153:391-7.

11 Zeiher BG, Artigas A, Vincent JL, et al. Neutrophil elastase inhibition in acute lung injury: results of the STRIVE Study. Crit Care Med 2004;32:1695-702.

12 Perkins GD, McAuley DF, Richter A, et al. Bench-to-bedside review: $\beta 2$-agonists and the acute respiratory distress syndrome. Crit Care 2004;8:25-32.

13 Dhingra VK, Uusaro A, Holmes $\mathrm{CL}$, et al. Attenuation of lung inflammation by adrenergic agonists in murine acute lung injury. Anesthesiology 2001;95:947-53.

14 Wu CC, Liao MH, Chen SJ, et al. Terbutaline prevents circulatory failure and mitigates mortality in rodents with endotoxemia. Shock 2000;14:60-7.

15 Braga PC, Mancini L, Guffanti EE, et al. Effects of nedocromil sodium on the oxidative burst of polymorphonuclear leukocytes: comparison with salbutamol. Drugs Exp Clin Res 1997;23:33-8.

16 Opdahl H, Benestad HB, Nicolaysen G. Effect of $\beta$-adrenergic agents on human neutrophil granulocyte activation with $\mathrm{N}$-formyl-methionyl-leucyl-phenylalanine and phorbol myristate acetate. Pharmacol Toxicol 1993;72:221-8.

17 van der Poll T, Calvano SE, Kumar A, et al. Epinephrine attenuates downregulation of monocyte tumor necrosis factor receptors during human endotoxemia. J Leukoc Biol 1997;61:156-60.

18 Maris NA, de Vos AF, Dessing MC, et al. Antiinflammatory effects of salmeterol after inhalation of lipopolysaccharide by healthy volunteers. Am J Respir Crit Care Med 2005; 172:878-84.

19 Perkins GD, McAuley DF, Thickett DR, et al. The $\beta$-agonist lung injury trial (BALTI): a randomized placebo-controlled clinical trial. Am J Respir Crit Care Med 2006; 173:281-7.

20 Jepsen LV, Skottun T. A rapid one-step method for the isolation of human granulocytes from whole blood. Scand J Clin Lab Invest 1982;42:235-8.

21 Nelson RD, Herron MJ. Agarose method for human neutrophil chemotaxis. Methods Enzymol 1988;162:50-9.

22 Bernard GR, Artigas A, Brigham KL, et al. Report of the American-European Consensus conference on acute respiratory distress syndrome: definitions, mechanisms, relevant outcomes, and clinical trial coordination. Consensus Committee. J Crit Care 1994;9:72-81.

23 Perkins GD, Chatteriie S, McAuley DF, et al. Role of nonbronchoscopic lavage for investigating alveolar inflammation and permeability in acute respiratory distress syndrome. Crit Care Med 2006;34:57-64.
24 Gunning K Rowan K. ABC of intensive care: outcome data and scoring systems. BMJ 1999:319:241-4.

25 Katzenelson R, Perel A, Berkenstadt $\mathrm{H}$, et al. Accuracy of transpulmonary thermodilution versus gravimetric measurement of extravascular lung water. Crit Care Med 2004;32:1550-4

26 Bateman J, Parida SK, Nash GB. Neutrophil integrin assay for clinical studies. Cell Biochem Funct 1993;11:87-91.

27 McAuley DF, Frank JA, Fang X, et al. Clinically relevant concentrations of $\beta 2-$ adrenergic agonists stimulate maximal cyclic adenosine monophosphatedependent airspace fluid clearance and decrease pulmonary edema in experimental acid-induced lung injury. Crit Care Med 2004;32:1470-6.

28 Harvath L, Robbins JD, Russell AA, et al. cAMP and human neutrophil chemotaxis. Elevation of cAMP differentially affects chemotactic responsiveness. $J$ Immunol 1991;146:224-32.

29 Silvestri M, Oddera S, Lantero S, et al. Beta 2-agonist-induced inhibition of neutrophil chemotaxis is not associated with modification of LFA-1 and Mac-1 expression or with impairment of polymorphonuclear leukocyte antibacterial activity. Respir Med 1999;93:416-23.

30 Llewellyn-Jones CG, Stockley RA. The effects of $\beta$ 2-agonists and methylxanthines on neutrophil function in vitro. Eur Respir J 1994:7:1460-6.

31 Doerschuk CM. The role of CD18-mediated adhesion in neutrophil sequestration induced by infusion of activated plasma in rabbits. Am J Respir Cell Mol Biol 1992; 7:140-8.

32 Kubo H, Graham L, Doyle NA, et al. Complement fragment-induced release of neutrophils from bone marrow and sequestration within pulmonary capillaries in rabbits. Blood 1998;92:283-90

33 Skoutelis AT, Kaleridis V, Athanassiou GM, et al. Neutrophil deformability in patients with sepsis, septic shock, and adult respiratory distress syndrome. Crit Care Med 2000;28:2355-9.

34 Doerschuk CM, Mizgerd JP, Kubo H, et al. Adhesion molecules and cellular biomechanical changes in acute lung injury: Giles F. Filley Lecture. Chest 1999;116:S37-43.

35 Masclans JR, Barbera JA, MacNee W, et al. Salbutamol reduces pulmonary neutrophil sequestration of platelet-activating factor in humans. Am J Respir Crit Care Med 1996;154:529-32.

36 Derian CK, Santulli RJ, Rao PE, et al. Inhibition of chemotactic peptide-induced neutrophil adhesion to vascular endothelium by cAMP modulators. J Immunol 1995:154:308-17

37 Altenburg SP, Bozza PT, Martins MA, et al. Adrenergic modulation of the blood neutrophilia induced by platelet activating factor in rats. Eur J Pharmacol 1994;256:45-9.

38 Rainer TH. L-selectin in health and disease. Resuscitation 2002:52:127-41.

39 Lee WL, Downey GP. Neutrophil activation and acute lung injury. Curr Opin Crit Care 2001;7:1-7.

40 Mills PJ, Goebel M, Rehman J, et al. Leukocyte adhesion molecule expression and T cell naive/memory status following isoproterenol infusion. J Neuroimmuno 2000;102: 137-44. 